\title{
Estado nutricional del paciente en hemodiálisis y factores asociados
}

\author{
Lídia Gómez Vilaseca, Mónica Manresa Traguany, Josefina Morales Zambrano, Elena García Monge, María \\ José Robles Gea, Julio Leonel Chevarria Montesinos
}

Unidad de diálisis. Hospital de Palamós. Gerona. España

\section{Resumen}

Introducción: La desnutrición en pacientes crónicos en diálisis tiene una alta prevalencia e importante repercusión en la morbimortalidad. Dado que no existen estudios previos en nuestro servicio, planteamos un estudio cuyo objetivo es evaluar el estado nutricional y los factores asociados.

Material y Método: Estudio descriptivo, observacional, transversal, el segundo trimestre de 2015 , en los pacientes en programa de hemodiálisis de un hospital comarcal. Se registró la edad, sexo, I. de Charlson, técnica de diálisis, IMC, tiempo en diálisis, albúmina, $\mathrm{PCR}$, colesterol y transferrina séricas. Se utilizó como instrumento la Valoración global subjetiva y el Score Malnutrición Inflamación.

Resultados: Se analizaron 35 pacientes, la edad media fue 72,2 años (DS: 11,8), 34,3\% fueron mujeres, el IMC es 27,1 (DS: 4,9), I. de Charlson 6,4 (DS: 1,7 ), $77,1 \%$ hacían diálisis convencional y 22,9\% hemodiafiltración en línea. Según el test MIS el $46 \%$ tenía buen estado nutricional y $54 \%$ estaba mal nutrido. Según la VGS $66 \%$ tenía buen estado nutricional, 31\% riesgo de desnutrición y 3\% desnutrición severa.

La VGS se relaciona con el IMC ( $p: 0,02$ ), creatinina (p: 0,001$)$, colesterol total ( $p: 0,02)$ y PCR (p: 0,01$)$; no con la edad, tiempo en hemodiálisis, I. Charlson, transferrina y albúmina $(p>0,05)$.

El Score Malnutrición Inflamación (MIS) se relaciona con el IMC ( $p: 0,002)$, I. Charlson ( $p: 0,01)$, creatinina

Correspondencia:

Lídia Gómez Vilaseca

Hospital de Palamós

Calle Hospital 17-19, Planta baja, Edificio Fleming

17230 Palamós. Gerona

E-mail: Igomez@ssibe.cat
( $p: 0,009)$ y PCR ( $p: 0,02)$; no con la edad, tiempo en hemodiálisis, colesterol total, transferrina y albúmina $(p>0,05)$.

Conclusión: Existe buena correlación entre las dos herramientas y los parámetros analíticos utilizados. Los pacientes en hemodiafiltración en línea tienen buen estado nutricional. No existe un único parámetro que valore la nutrición. Se sugiere que es necesaria la valoración nutricional dada la alta prevalencia que existe.

PALABRAS CLAVE: nutrición; hemodiálisis; VGS; inflamación; desnutrición.

Nutritional status of the patient on hemodialysis and associated factors

\begin{abstract}
Introduction: Malnutrition in chronic dialysis patients has a high prevalence and important repercussion in morbidity and mortality. Since there are no previous studies in our service, we propose a study whose objective is to evaluate the nutritional status and associated factors.
\end{abstract}

Material and Method: A descriptive, observational, cross-sectional study, in the second trimester of 2015, in patients in the hemodialysis program of a county hospital. Age, sex, Charlson's index, dialysis technique, BMI, time on dialysis, albumin, CRP, cholesterol and serum transferrin were recorded. Subjective global assessment and Score Malnutrition Inflammation were used as instrument. 
Results: We analyzed 35 patients, mean age was 72.2 years (SD: 11.8$), 34.3 \%$ were women, BMI was 27.1 (SD: 4.9), Charlson index 6.4 (DS: 1.7), $77.1 \%$ were on conventional dialysis and $22.9 \%$ were on-line hemodiafiltration. Regarding the MIS test, $46 \%$ had good nutritional status and $54 \%$ were malnourished. According to the VGS, $66 \%$ had good nutritional status, $31 \%$ risk of malnutrition and $3 \%$ severe malnutrition.

VGS was related to BMI (p: 0.02), creatinine ( $p$ : 0.001 ), total cholesterol (p: 0.02), and CRP (p: 0.01); and it was not related to age, time on hemodialysis, Charlson's index, transferrin and albumin ( $p>0.05$ ).

The Score Malnutrition Inflammation (MIS) was related to BMI ( $p: 0.002$ ), Charlson's index (p: 0.01), creatinine ( $p: 0.009)$ and CRP ( $p: 0.02)$; not with age, time on hemodialysis, total cholesterol, transferrin and albumin ( $p>0.05$ ).

Conclusions: There is good correlation between the two tools and analytical parameters used. Patients in on-line hemodiafiltration have good nutritional status. There is no single parameter that evaluate nutrition. The inclusion of nutritional assessment is suggested given the high prevalence.

KEYWORDS: nutrition; hemodialysis;VGS; inflammation; malnutrition.

\section{Introducción}

Existe una alta prevalencia de desnutrición en pacientes con insuficiencia renal, sobre todo al inicio de diálisis, con repercusión en la morbimortalidad, e implica aumento de ingresos hospitalarios, estancia media, número de infecciones y mortalidad fundamentalmente por causas cardiovasculares.

La mortalidad cardiovascular se relaciona con la alta prevalencia de factores de riesgo, sumado al efecto nocivo de la reacción inflamatoria subyacente en el endotelio, que comporta ateromatosis acelerada y al que se ha nombrado como MIA (malnutrición, inflamación, ateromatosis $)^{1,2}$. La biocompatibilidad del sistema de diálisis se relaciona con la inflamación sistémica, esto sumado a los procesos intercurrentes como infecciones agudas y/o crónicas, comportan un estado catabólico mantenido que favorece la desnutrición ${ }^{3,4}$.
Los factores determinantes de la desnutrición se observan en la enfermedad renal crónica; como la anorexia, trastornos digestivos, comorbilidad asociada, alteraciones hormonales, acidosis metabólica, entorno urémico, dietas no controladas o la disminución de la ingesta, que es probablemente la más importante ${ }^{5}$. Diferentes estudios observan signos de desnutrición en pacientes de hemodiálisis y van de $23-76 \%{ }^{6,7}$. El estudio DOPPS ${ }^{8}$ que se realizó en siete países (Estados unidos, Japón, Francia, Alemania, Reino unido, Italia y España), encontró desnutrición moderada-severa en 18,9\% según la VGS (valoración global subjetiva) ${ }^{9}$.

Una forma de prevenir la malnutrición es identificar aquellos pacientes que se encuentran con riesgo nutricional, por este motivo planteamos un estudio cuyo objetivo es evaluar el estado nutricional de los pacientes de diálisis en nuestra unidad y determinar los factores asociados. Como objetivo secundario se analizó el estado de nutrición en función de las técnicas de hemodiálisis.

\section{Material y Método}

Estudio descriptivo observacional de corte transversal, realizado el segundo trimestre del 2015, en la unidad de diálisis de un hospital comarcal. La población diana fueron los 44 pacientes en programa de hemodiálisis, seleccionando como población de estudio los que llevaban al menos un mes de tratamiento y mayores de 18 años, que aceptaron participar y firmaron el consentimiento informado. Se excluyeron los pacientes hospitalizados en el momento del estudio y aquellos con patología psiquiátrica o incapacidad para responder el cuestionario.

El estado de inflamación-malnutrición se evaluó mediante el test MIS ${ }^{10}$ (Score Malnutrición Inflamación) con una puntuación de 0-3011, además si era $\geq 0$ ó $<10$. El grado de desnutrición se evaluó mediante el instrumento VGS ${ }^{12}$.

Como variables secundarias se registraron: edad, sexo, I. de Charlson ${ }^{13}$ técnica de diálisis (Hemodiafiltración en línea (HDF) y hemodiálisis convencional (HD)), IMC, tiempo de diálisis, albúmina, creatinina, $P C R$, colesterol y transferrina sérica. Los datos se obtuvieron de la historia clínica electrónica.

Para el análisis estadístico se utilizó el programa SPSS19, las variables cualitativas se expresaron como frecuencias y porcentajes, las cuantitativas mediante 
media, mediana y sus rangos. Para el análisis bivariable, se utilizaron los test de $X^{2}$, $t$ de student o Rho de Spearman según correspondía, se evaluaron los OR e IC al 95\%, se consideró significativo una $p<0,05$.

Se pidió consentimiento informado a los participantes, los datos se trataron de forma confidencial y el proyecto fue aprobado por el comité de investigación del centro.

\section{Resultados}

Se analizaron 35 pacientes, 34,3\% eran mujeres y $65,7 \%$ hombres. El $77,1 \%$ realizaban HD y $22,9 \%$ HDF. Las características generales de los pacientes se pueden ver en la Tabla 1.

Tabla 1. Características generales de la población en estudio.

\begin{tabular}{|lcc|}
\hline Variables & Media & DE \\
\hline Edad (años) & 72,18 & 11,85 \\
\hline Tiempo HD (meses) & 47,34 & 34,49 \\
\hline IMC $\left(\mathrm{Kg} / \mathrm{m}^{2}\right)$ & 27,12 & 4,92 \\
\hline I. Charlson & 6,44 & 1,77 \\
\hline Creatinina (mg/dl) & 7,59 & 2,7 \\
\hline Colesterol total (mg/dl) & 137,06 & 31,26 \\
\hline Transferrina (mg/dl) & 156,34 & 29,42 \\
\hline Albúmina (mg/dl) & 3,65 & 0,39 \\
\hline PCR (mg/dl) & 7,50 & 9,1 \\
\hline
\end{tabular}

Las características de los pacientes según las diferentes técnicas de diálisis se observan en la Tabla 2.
Tabla 2. Comparación de los pacientes en diálisis según la técnica.

\begin{tabular}{lccc}
\hline & HD & HDF & p \\
\cline { 1 - 2 } Edad ( años) & 74,1 (DS 10,5) & 64,5 (DS 13,9) & 0,04 \\
\hline IMC (kg/m²) & 26,5 (DS 4,8) & 29,1 (DS 5,0) & 0,20 \\
\hline I. Charlson & 6,9 (DS 1,5) & 4,7 (DS 1,4) & 0,001 \\
\hline \multicolumn{2}{l}{ Tiempo en HD (meses) 42,5 (DS 34,1) } & 60,4 (DS 31,2) & 0,18 \\
\hline
\end{tabular}

Respecto a la comparación de pacientes según la técnica de diálisis realizada resultó estadísticamente significativa la Edad (74,1 años en HD y 64,5 años en diálisis HDF, $p=0,04)$ y también el I.Charlson $(6,9$ vs $4,7 p=$ $0,001)$, no hubo relación estadísticamente significativa con el IMC, ni el tiempo en hemodiálisis.

La valoración con el test VGS se observa en el gráfico $\mathbf{l}$.

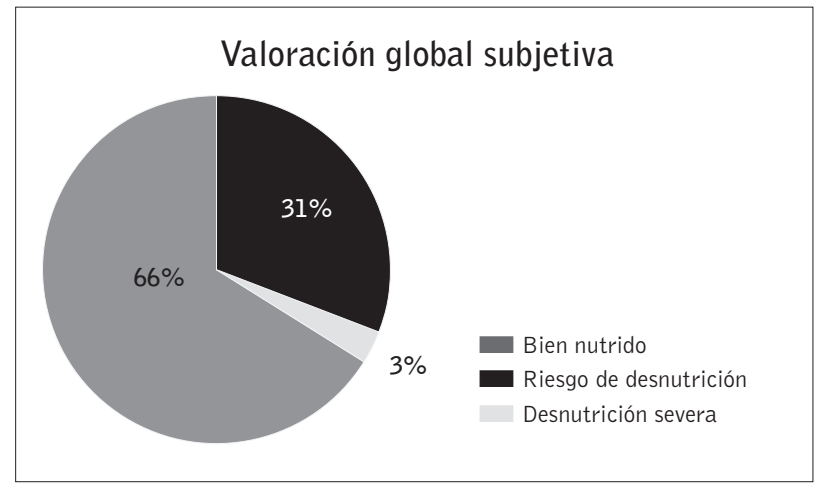

Gráfico 1. Resultados del test VGS en los pacientes en diálisis.

El grupo con riesgo de desnutrición o desnutrición moderada y desnutrición severa se consideró como uno solo.

Tabla 3. Relación entre las variables analizadas y la VGS.

\begin{tabular}{|lccccc|}
\hline Factores & Bien nutrido & (DS) & Riesgo de desnutrición & (DS) & $\mathbf{p}$ \\
\hline Edad (años) & 71,04 & 12,99 & 74,41 & 9,43 & 0,619 \\
\hline Tiempo HD (meses) & 48,24 & 33,36 & 45,36 & 37,92 & 0,74 \\
\hline IMC (kg/m2) & 28,54 & 4,68 & 24,4 & 4,33 & 1,5 \\
\hline I. Charlson & 5,99 & 5,99 & 7,31 & 1,88 & 0,092 \\
\hline Creatinina (mg/dl) & 8,54 & 2,63 & 5,77 & 25,86 & 0,001 \\
\hline Colesterol (mg/dl) & 145,96 & 30,35 & 120 & 23,9 & 0,344 \\
\hline Transferrina (mg/dl) & 160,7 & 31,54 & 148 & 0,35 & 0,344 \\
\hline Albúmina (mg/dl) & 3,69 & 0,41 & 3,56 & 12,48 \\
\hline PCR ( $\mathrm{mg} / \mathrm{dl})$ & 4,37 & 4,58 & 13,5 & 0,013 \\
\hline
\end{tabular}


Existe relación entre el riesgo alto de desnutrición según la VGS y la técnica de HD (RP 1,48, IC95\%: 1,08 a 2,$04 ; p=0,032 ;$ ) siendo la diálisis convencional la de referencia, mas no con el sexo (OR 1,92; IC95\% 0,4 $-9,1 ; p=0,4)$.

Respecto a la relación del estado nutricional de los pacientes, medido por la VGS, con los otros factores analizados en el el estudio, resultaron significativos el IMC (28,54 en bien nutridos y el 24,4 en los que tenían riesgo de desnutrición; $p=0,023)$, creatinina ( 8,54 vs. 5,77; $p=0,001)$, colesterol ( 145,96 vs. 120,$00 ; p=0,021$ ) y $\operatorname{PCR}(4,37$ vs. 13,$5 ; p=0,013)$. No hubo relación significativa con la edad, tiempo en hemodiálisis, índice de Charlson, transferrina ni albúmina. (Tabla 3).

Mediante el test MIS se obtuvo una media de 9.83 (IC95\% 8,3 -11,3, DS 4.27), el 45,8\% presentaba una puntuación $\geq 10$. (Gráfico 2).

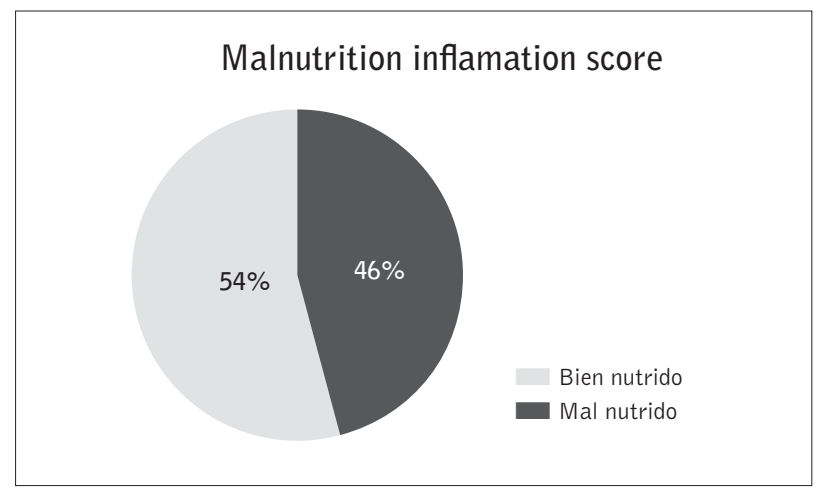

Gráfico 2. Resultados del test MIS en los pacientes en diálisis.

No se observa relación entre el riesgo de desnutrición según el test MIS con la técnica de diálisis $(0 R 0,11$; IC $95 \% 0,01-1,06 ; p=0,059$ ) y con el sexo (IC $95 \%$ $0,8-18,23 ; 0 \mathrm{R} 3,9 ; \mathrm{p}: 0,15)$, las demás relaciones se observan en la tabla 4 y tabla 5.

Tabla 4. Relación entre las variables analizadas y y el MIS $\geq 10$ (categórico).

\begin{tabular}{|lccccc}
\hline Factores & Bien nutrido & (DS) & Riesgo de desnutrición & (DS) & $\mathbf{p}$ \\
\hline Edad (años) & 69,15 & 13,31 & 77,48 & 8,97 & 0,217 \\
\hline Tiempo HD (meses) & 47,42 & 36,55 & 47,24 & 33,06 & 0,90 \\
\hline IMC (kg/m2) & 29,46 & 4,76 & 24,39 & 3,61 & 1,32 \\
\hline I. Charlson & 4,71 & 1,80 & 7,31 & 2,12 & 0,002 \\
\hline Creatinina (mg/dl) & 8,67 & 2,74 & 6,30 & 27,50 & 0,142 \\
\hline Colesterol (mg/dl) & 144,21 & 32,88 & 128,56 & 20,56 & 0,142 \\
\hline Transferrina (mg/dl) & 164,53 & 33,59 & 146,63 & 0,330 & 0,832 \\
\hline Albúmina (mg/dl) & 3,66 & 0,44 & 3,64 & 11,50 \\
\hline PCR (mg/dl) & 4,116 & 4,55 & 11,51 & 0,020 \\
\hline
\end{tabular}

Tabla 5. Relación entre las variables analizadas y el MIS de 0-30.

\begin{tabular}{|c|c|c|}
\hline Factores & $\mathbf{R}$ & $\mathbf{P}$ \\
\hline \multicolumn{2}{|l|}{ Sexo (mujer/varón) } & 0,068 \\
\hline \multicolumn{2}{|c|}{ Técnica de HD (Convencional/On-line) } & 0,006 \\
\hline Edad (años) & $r: 0,37$ & $p: 0,03$ \\
\hline Tiempo HD (meses) & $r: 0,10$ & $\mathrm{p}: 0,57$ \\
\hline IMC $(\mathrm{kg} / \mathrm{m} 2)$ & $r:-0,56$ & $p: 0,001$ \\
\hline I. Charlson & $r: 0,52$ & $\mathrm{p}: 0,001$ \\
\hline Creatinina (mg/dl) & $r:-0,57$ & $\mathrm{p}: 0,001$ \\
\hline Colesterol (mg/dl) & $r:-0,29$ & $\mathrm{p}: 0,08$ \\
\hline Transferrina (mg/dl) & $r:-3,75$ & p: 0,27 \\
\hline Albúmina (mg/dl) & $r:-0,11$ & $\mathrm{p}: 0,53$ \\
\hline PCR (mg/dl) & $r: 0,50$ & $p: 0,002$ \\
\hline
\end{tabular}

\section{Discusión y conclusiones}

Nuestra muestra de estudio tiene una edad media (72 años), que se corresponde a la media nacional de pacientes en $\mathrm{HD}^{14}$, tienen un tiempo en HD de casi 4 años, alta comorbilidad y sobrepeso.

Según el test de VGS y MIS existe una alta prevalencia de pacientes con riesgo de desnutrición y/o desnutridos en nuestro centro, que se corresponde con los trabajos de Carrascal $\mathrm{S}$ y cols ${ }^{15}$ y Cansino y cols dónde presentan una prevalencia entre el $20-50 \%$ de malnutrición proteico-calórica e inflamación ${ }^{16}$.

Hemos observado que los test VGS y MIS se correlacionan bien con el IMC y el I. Charlson, mostrando que a mayor comorbilidad es mayor el riesgo de desnutri- 
ción, y que un mejor estado nutricional se relaciona con un IMC mayor. No existe relación con el tiempo en hemodiálisis y la edad.

Las herramientas MIS y VGS se correlacionan bien con los parámetros bioquímicos de desnutrición, la VGS con la creatinina, colesterol y PCR, y el test MIS con la creatinina y PCR, y claramente tendencia a tener valores inferiores en colesterol y transferrina. Sin embargo ninguno de los dos con la albúmina.

Según estudios previos los valores altos de creatinina, colesterol, transferrina ${ }^{17}$ y albúmina se correlacionan con una mejor ingesta; en nuestro estudio es claramente significativo el valor de creatinina, sin embargo el de albúmina no ha variado, lo que puede reflejar que en ocasiones la albúmina no sea un marcador fiable ${ }^{18,19}$ y otros como la prealbúmina tengan una mejor correlación ${ }^{20}$.

Los pacientes en HDF tienen un $0 \%$ de desnutrición, siendo estadísticamente significativo, lo cual es muy alentador. La técnica HDF se ha asociado a menor inflamación, eliminación más eficiente de las toxinas urémicas, una incidencia menor de eventos cardiovasculares $^{21}$ y reducción de la mortalidad en comparación con la HD22, sobre todo en pacientes ancianos, no diabéticos y con mayor comorbilidad. Sin embargo en metaanálisis recientes no queda una clara evidencia de que la técnica on-line mejore el pronóstico de los pacientes ${ }^{23-25}$. En nuestro estudio los pacientes en HDF eran pocos, más jóvenes, clínicamente estables y con menor comorbilidad, lo cual puede inducir un sesgo considerable, por lo que es necesario realizar estudios con un mayor número de pacientes y con grupos más comparables.

La mayoría de las guías recomiendan la realización de encuestas dietéticas periódicas en los pacientes de diálisis, ya que el deterioro del estado nutricional es indicativo de intervención terapéutica ${ }^{26,27}$. En nuestro hospital se realiza una vez iniciado el tratamiento de diálisis y en el programa de crónicos solo en casos que se detectan alteraciones, como podría ser un mal cumplimento de la dieta prescrita.

Dado que el número de pacientes que tenemos en riesgo de desnutrición es alto, sería preciso instaurar un seguimiento de los aspectos nutricionales y realizar una intervención personalizada para intentar revertir la situación actual.

\section{Agradecimientos}

Especialmente al doctor Julio Chevarría, por su gran ayuda, al comité de recerca del Hospital de Palamós por la colaboración en este trabajo, a todas mis compañeras y la unidad de Nefrología del Hospital de Palamós.

$$
\begin{gathered}
\text { Recibido: } 29 \text { agosto } 2016 \\
\text { Revisado: } 5 \text { octubre } 2016 \\
\text { Modificado: } 15 \text { diciembre } 2016 \\
\text { Aceptado: } 25 \text { enero } 2017
\end{gathered}
$$

\section{Bibliografía}

1. Bistrian BR. Role of the systemic inflammatory response syndrome in the development of proteincalorie malnutrition in ESRD. Am J Kidney Dis Off J Natl Kidney Found. 1998 Dec;32(6 Suppl 4):S113-7.

2. Riella MC. Malnutrition in dialysis: malnourishment or uremic inflammatory response? Kidney Int. 2000 Mar;57(3):1211-32.

3. Ayus JC, Sheikh-Hamad D. Silent infection in clotted hemodialysis access grafts. J Am Soc Nephrol JASN. 1998 Jul;9(7):1314-7.

4. Schindler R, Boenisch 0, Fischer C, Frei U. Effect of the hemodialysis membrane on the inflammatory reaction in vivo. Clin Nephrol. 2000 Jun;53(6):452-9.

5. Hakim RM, Levin N. Malnutrition in hemodialysis patients. Am J Kidney Dis Off J Natl Kidney Found. 1993 Feb;21(2):125-37.

6. Qureshi AR, Alvestrand A, Danielsson A, DivinoFilho JC, Gutierrez A, Lindholm B, et al. Factors predicting malnutrition in hemodialysis patients: a cross-sectional study. Kidney Int. 1998 Mar;53(3):773-82.

7. Marckmann P. Nutritional status of patients on hemodialysis and peritoneal dialysis. Clin Nephrol. 1988 Feb;29(2):75-8.

8. Young EW, Goodkin DA, Mapes DL, Port FK, Keen $M L$, Chen $K$, et al. The Dialysis Outcomes and Practice Patterns Study (DOPPS): An international hemodialysis study. Kidney Int. 2000;57:S74-81. 
9. Hecking E, Bragg-Gresham JL, Rayner HC, Pisoni $\mathrm{RL}$, Andreucci VE, Combe C, et al. Haemodialysis prescription, adherence and nutritional indicators in five European countries: results from the Dialysis Outcomes and Practice Patterns Study (DOPPS). Nephrol Dial Transplant Off Publ Eur Dial Transpl Assoc - Eur Ren Assoc. 2004 Jan;19(1):100-7.

10. Kalantar-Zadeh K, Kopple JD, Block G, Humphreys MH.A malnutrition-inflammation score is correlated with morbidity and mortality in maintenance hemodialysis patients. Am J Kidney Dis Off $\mathrm{J}$ Natl Kidney Found. 2001 Dec;38(6):1251-63.

11. Carreras RB, Mengarelli MC, Najun-Zarazaga CJ. El score de desnutrición e inflamación como predictor de mortalidad en pacientes en hemodiálisis. Diálisis Traspl. 2008;29(02):55-61.

12. Enia G, Sicuso C, Alati G, Zoccali C. Subjective global assessment of nutrition in dialysis patients. Nephrol Dial Transplant Off Publ Eur Dial Transpl Assoc - Eur Ren Assoc. 1993;8(10):1094-8.

13. Charlson ME, Pompei P, Ales KL, MacKenzie CR. A new method of classifying prognostic comorbidity in longitudinal studies: development and validation. J Chronic Dis. 1987;40(5):373-83.

14. Martín Escobar E, Registro Español de Enfermos Renales (REER). The Spanish Renal Registry: 2013 report and evolution from 2007-2013. Nefrol Publ Of Soc Esp Nefrol. 2016;36(2):97-120.

15. Elvira Carrascal S, Colomer Codinachs $M$, Pérez Oller L, Chirveches Pérez E, Puigoriol Juvanteny $E$, Pajares Requena $D$, et al. Descripción del estado nutricional de los pacientes de una unidad de diálisis mediante el uso de la escala "Malnutrition Inflamation Score." Enferm Nefrológica. 2013 Mar;16(1):23-30.

16. Cansino J, Carmen M, Amezquita Orjuela Y, Sánchez Márquez V, Sánchez Fernández C, Fernández de la Vega $E$, et al. Utilidad del Score Malnutrición Inflamación (SMI) en la evaluación nutricional de los pacientes en hemodiálisis. Enferm Nefrológica. 2012 Jan;15:80-80.

17. Carrero JJ, Chen J, Kovesdy CP, Kalantar-Zadeh K. Critical appraisal of biomarkers of dietary intake and nutritional status in patients undergoing dialysis. Semin Dial. 2014 Dec;27(6):586-9.

18. Ikizler TA. The use and misuse of serum albumin as a nutritional marker in kidney disease. Clin J Am Soc Nephrol CJASN. 2012 Sep;7(9):1375-7.
19. Kuzuya M, Izawa S, Enoki H, Okada K, Iguchi A. Is serum albumin a good marker for malnutrition in the physically impaired elderly? Clin Nutr Edinb Scotl. 2007 Feb;26(1):84-90.

20. Beck FK, Rosenthal TC. Prealbumin: a marker for nutritional evaluation. Am Fam Physician. 2002 Apr 15;65(8):1575-8.

21. Tellingen AV, Grooteman MP, Bartels $P C$, Limbeek JV, Guldener CV, Wee PMT, et al. Long-term reduction of plasma homocysteine levels by superflux dialyzers in hemodialysis patients. Kidney Int. 2001;59(1):342-7.

22. Maduell F, Moreso F, Pons M, Ramos R, MoraMacia J, Carreras J, et al. High-Efficiency Postdilution Online Hemodiafiltration Reduces AllCause Mortality in Hemodialysis Patients. J Am Soc Nephrol. 2013 Mar 1;24(3):487-97.

23. Wang AY, Ninomiya T, Al-Kahwa A, Perkovic V, Gallagher MP, Hawley C, et al. Effect of Hemodiafiltration or Hemofiltration Compared With Hemodialysis on Mortality and Cardiovascular Disease in Chronic Kidney Failure: A Systematic Review and Meta-analysis of Randomized Trials. Am J Kidney Dis. 2014 Jun;63(6):968-78.

24. Nistor I, Palmer SC, Craig JC, Saglimbene V, Vecchio $M$, Covic $A$, et al. Haemodiafiltration, haemofiltration and haemodialysis for end-stage kidney disease. Cochrane Database Syst Rev. 2015;5:CD006258.

25. Mostovaya IM, Blankestijn PJ, Bots ML, Covic A, Davenport A, Grooteman MPC, et al. Clinical evidence on hemodiafiltration: a systematic review and a meta-analysis. Semin Dial. 2014 Mar;27(2):119-27.

26. Ruperto López M, Barril Cuadrado G, Lorenzo Sellares $V$. [Nutrition guidelines for advanced chronic kidney disease (ACKD)]. Nefrol Publ of Soc Esp Nefrol. 2008;28 Suppl 3:79-86.

27. Huarte-Loza (coordinadora) E, Barril-Cuadrado G, Cebollada-Muro J, Cerezo-Morales S, CoronelDíaz $F$, Doñate-Cubells $T$, et al. Nutrición en pacientes en diálisis. Consenso SEDYT. Diálisis Traspl. 2006;27(04):138-61. 